\title{
On Multistability of Delayed Genetic Regulatory Networks with Multivariable Regulation Functions
}

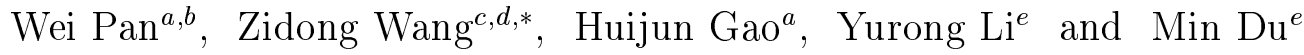

\begin{abstract}
Many genetic regulatory networks (GRNs) have the capacity to reach different stable states. This capacity is defined as multistability which is an important regulation mechanism. Multiple time-delays and multivariable regulation functions are usually inevitable in such GRNs. In this paper, multistability of GRNs is analyzed by applying the control theory and mathematical tools. This study is to provide a theoretical tool to facilitate the design of synthetic gene circuit with multistability in the perspective of control theory. By transforming such GRNs into a new and uniform mathematical formulation, we put forward a general sector-like regulation function that is capable of quantifying the regulation effects in a more precise way. By resorting to up-to-date techniques, a novel Lyapunov-Krasovskii functional (LKF) is introduced for achieving delay dependence to ensure less conservatism. New conditions are then proposed to ensure the multistability of a GRN in the form of linear matrix inequalities (LMIs) that are dependent on the delays. Our multistability conditions are applicable to several frequently used regulation functions especially the multivariable ones. Two examples are employed to illustrate the applicability and usefulness of the developed theoretical results.
\end{abstract}

\section{Keywords}

Multistability, multivariable regulation function, genetic regulatory networks, Lyapunov-Krasovskii functional, linear matrix inequality, multiple time-delays.

\section{INTRODUCTION}

Systems biology is the study of an organism, viewed as an integrated and interacting network of genes, proteins and biochemical reactions which give rise to life, instead of analyzing individual components or aspects of the organism [1-3]. The focus on systems as opposed to individual genes or pathways is shared by the contemporaneous discipline of systems biology, which analyzes biological organisms in their entirety [4-6]. The spirit of genetic engineering in which genes and gene products are considered as a whole system could be extended to synthetic biology. In synthetic biology, the ultimate goal is to engineer unnatural biological systems that function in living organism to investigate natural biological phenomena for a variety of applications. It is reasonable to expect that ideas and method from systems and control theory which is powerful in analyzing dynamical properties and designing controller to achieve desired performance will lead to new understanding of the underlying biological processes therefore having potential applications in designing synthetic gene circuit.

This work was supported in part by the Biotechnology and Biological Sciences Research Council (BBSRC) of the U.K. under Grants BB/C506264/1 and 100/EGM17735, the Royal Society of the U.K., the National Natural Science Foundation of China under Grant 61028008, and the International Science and Technology Cooperation Project of China under Grant 2009DFA32050.

${ }^{a}$ Space Control and Inertial Technology Research Center, Harbin Institute of Technology, Harbin 150001, China.

${ }^{b}$ Department of Electronic Science and Technology, University of Science and Technology of China, Hefei 230027, China.

${ }^{c}$ School of Information Science and Technology, Donghua University, Shanghai 200051, China.

${ }^{d}$ Department of Information Systems and Computing, Brunel University, Uxbridge, Middlesex, UB8 3PH, United Kingdom.

${ }^{e}$ Department of Electrical Engineering, Fuzhou University, Fuzhou 350002, China.

${ }^{*}$ Corresponding author. E-mail: Zidong.Wang@brunel.ac.uk 
The synthetic genetic regulatory networks (GRNs) prove to be a powerful tool in studying gene regulation processes in living organisms [7-12]. By using ordinary differential equations to describe the rates of concentration change in biochemical substance, such as genes, proteins, activators, repressors, enzymes, factors or products of a biochemical network, more detailed understanding and insights of the dynamic behavior exhibited by biological systems can be explored [13,14]. In particular, since GRNs are high-dimensional and nonlinear, it is also indispensable to consider the network dynamics from the viewpoint of systems and control theory [15-17].

Obviously, the precise structure of a mathematical model should be consistent with the dynamical behaviors of the system. It has been recognized that the slow processes of transcription, translation and diffusion to the place of action of a protein inevitably cause time delays. Also, for different substance in GRNs, time delays of biochemical reactions may vary due to the different reaction pathways. Time delays are frequently encountered in many other practical engineering systems besides GRNs, such as communication, electronics, and chemical systems. Therefore, in order to have more accurate models, it is necessary to take time-delays into account in GRNs. In the past decade, stability analysis and synthesis problems for various time-delay control systems have gained considerable research interests and a large amount of results have appeared in the literature, see, e.g. [18-21].

On the other hand, the regulation functions, which are either linear or nonlinear, play a crucial role in determining qualitative properties of GRNs, such as the number and the stability of steady states. The regulatory mechanism are actually descriptions of biochemical reaction kinetics law such as mass action law, Hill law, Henri-Michaelis-Menten law, etc. The linear or nonlinear regulation function are often single-variable and has a form of monotonicity with the single variable. Then the regulation functions for different substance in GRNs add together to regulate a certain kind. In synthetic GRNs, one of the simplest ways to implement such an additive input function is to provide a gene with multiple promoters, each responding to one of the inputs. However, to describe the complicated relationship between different biochemical substance in GRNs, it is natural to introduce multivariable regulation functions, in which different variables are multiplying or coupling together. GRNs with regulation functions of such forms have been reported in [22-24]. It should be mentioned that, even though there are no multivariable terms, the types of regulation functions could be diverse, most of which are nonlinear $[13,14]$.

Due to the nonlinearity of regulation functions, the coexistence of multiple steady states which refers to multistability is possible. The traditional notion of stability named as monostability in GRNs [25-27] is concerned with unique equilibrium point, and this differs significantly from the multistability mentioned here. It is noticed that multistability has certain properties which are not shared by other mechanisms of integrative control, therefore plays an important role in the dynamics of living cells and organisms [28-30]. For example, the maintenance of phenotypic differences in the absence of genetic or environmental differences, which has been demonstrated experimentally for the regulation of the lactose operon in Escherichia coli, may attribute to multistability. Cell differentiation might also be explained as multistability [31]. Bistability, a basic case of multistability, has a property that there are two stable fixed points. It has become increasingly clear that bistability is an important recurring theme in cell signaling and of particular relevance to biological systems that switch between discrete states, generate oscillatory responses. As stated in [32], bistability is a new way of looking at cell cycle control.

Recently, a lot of efforts have been made to the mathematical modeling of GRNs with multistability. In [8], a synthetic genetic co-repressive switches in the well known lac operon in the bacteria Escherichia coli were 
constructed and a model with two components was proposed. A more detailed mathematical model in which the parameters were all estimated from reported experimental data was developed in [22], and it was shown that there was bistability in the lactose operon dynamics for realistic extracellular lactose concentration values. A simplification of the above model that considered only the role of $\beta$-galactosidasein the operon regulation and ignores that of lactose permease, which also displayed bistability, was introduced in [23]. In [12], the dynamics of the bistable lactose utilization network of Escherichia coli has been quantitatively investigated in single cell experiments. In [24], Cdc2-Cyclin B/Wee1 system was transformed to a two-variable problem under necessary assumptions and displaying bistability.

Theoretical results obtained for the multistability of a GRN have been scattered in the literature. The biological system with multistability and hysteresis has been modeled as monotone dynamic systems in [33], where the rich and elegant theory of monotone dynamic system provides an efficient mathematical tool for analysis (see [34] and references therein). Especially, in the biological systems with bistability, each stable mode of operation is associated with an appropriate invariant set in the state space and stability with respect to each set has been studied in terms of a local notion of input-to-state stability with respect to compact sets [35]. In the control communities, stability analysis should always be performed prior to the controller design. On one hand, the results on multistability should facilitate the design of synthetic gene circuits with multistability while these results are difficult to extend to engineering. On the other hand, time-delays and multivariable regulation functions have not been considered in these results. How to analyze multistability of time-delay GRNs with multivariable regulation functions in order to be potential in synthetic biology remains as an open problem. Therefore, it is essential and important to investigate the multistability of delayed GRNs with multivariable regulation functions. In the survey paper [36], the trigger and significance of this study have been summarized for considering the possible control scheme for the switch of different phenotypes in terms of epigenetics. In [36], the essential roles of time delays, negative loop and positive loop have been thoroughly discussed. Time delays have a close relationship with oscillations, even with one element negative loops. In any case, a negative loop plays to generate homeostasis around a steady state located near the thresholds, while a positive loop is a necessary condition to generate multistationarity or a multiplicity of regimes in a more general way. Despite the importance of gaining straightforward insight on the cause of multistability, to the best of the authors' knowledge, there has been little effort towards the theoretical research on this challenging problem. Such a situation motivates our present study.

Lyapunov-Krasovskii functional (LKF) theory and linear matrix inequality (LMI) technique are powerful tools in stability analysis and controller design and have been extensively studied in the control communities (see [37-41] and references therein). Although there are also reports on the multistability analysis for neural networks $[42,43]$, these results are focusing on the analysis rather than aiming at design. Even in this community, there are seldom reports on multistability analysis by LKF and LMI. To facilitate the readers in biology area, let us briefly discuss the LKF theory and LMI technique. Lyapunov's direct method (also called the second method of Lyapunov) allows us to determine the stability of a system without explicitly solving differential equations. The method is a generalization of the idea that if there is some "measure of energy" in a system, then we can study the rate of change of the energy of the system to ascertain stability [44]. In case of systems with time-delay, such measure of energy is often adopted as the LKF, which is typically of the quadratic form. By calculating the derivative of the LKF, it is usually concluded that the overall time-delay system is stable if certain LMIs are feasible [37-41]. Note that the solvability of LMIs can be easily checked by using the Matlab toolbox, and a growing number of dynamics analysis problems can be converted into the 
feasibility of LMIs [45].

In this paper, we are concerned with the multistability of GRNs with multiple time-delays. Multivariable and several different types of regulation functions are considered. We then generalize the mathematical formulation of such GRNs by proposing a sector-like regulation function. A novel LKF is introduced and the most updated techniques are employed to achieve delay-dependence. A sufficient condition is then derived for the multistability of a GRN with multiple time delays and multivariable regulation functions in the form of LMIs. An important feature with the results to be reported is that, all the multistability conditions are dependent on the delays, made possible by utilizing the up-to-date techniques to achieve delay dependence. Second, our multistability conditions are applicable to several different regulation functions, which cover many types of currently investigated GRNs, especially including the complicated multivariable regulation functions. Two examples which have been tested by reported experiments are employed to illustrate the applicability and usefulness of the developed theoretical results. Example 1 is a Cdc2-Cyclin B/Wee1 system model and example 2 is a lactose system model. The GRNs in both of these examples display bistability.

Notation: The notation used throughout the paper is standard. The superscript $T$ indicates matrix transposition; $\mathbb{R}^{n}$ denotes the $n$-dimensional Euclidean space and $\mathbb{R}^{n \times k}$ is the set of all $n \times k$ real matrices. $A_{n, k}$ denotes $A \in \mathbb{R}^{n \times k} . I$ and $\mathbf{0}$ denote identity matrix and zero matrix respectively, the notation $P>0$ means that $P$ is symmetric and positive definite and the symbol $*$ indicates symmetric blocks in the LMIs. In addition, $\operatorname{diag}\{\ldots\}$ stands for a block-diagonal matrix and for a matrix $A, \operatorname{sym}(\mathrm{A}) \operatorname{denotes} A+A^{T}$.

\section{Model AND PRELIMinARIES}

In this section, we introduce a GRN model which can be described by the following differential equations for $i=1,2, \ldots, n$ :

$$
\dot{z}_{i}(t)=-a_{i} z_{i}(t)+\sum_{j=1}^{n} b_{i j} f_{i j}\left(z_{j}(t)\right)+\sum_{j=1}^{n} c_{i j} g_{i j}\left(z_{j}\left(t-\tau_{i}\right)\right)+\sum_{j=1}^{n} d_{i j} y_{i j}(t) h_{i j}\left(z_{j}(t)\right)+u_{i},
$$

where $z_{1}, \ldots, z_{n}$ are biochemical substance, such as genes, proteins, activators, repressors, enzymes, factors or products of a biochemical network, and $z(\cdot)=\left[z_{1}(\cdot), z_{2}(\cdot), \ldots, z_{n}(\cdot)\right]^{T} \in \mathbb{R}^{n}$ is the substance state vector. Their rates of degradation are denoted by $a_{i} \in R^{+}$. $\dot{z}_{i}$, the rate of change in $z_{i}$, represents concentration change of a variable due to production or degradation. $u_{i}$ is defined as a basal rate. $f_{i j}(\cdot)$ and $g_{i j}(\cdot)$ represent the feedback regulation function of the $j$ th substance on the $i$ th substance, which are generally nonlinear or linear single-variable functions.

Due to the fact that time delays occur in transcription, translation and diffusion to the place of action of a protein, and for different biochemical substance in GRNs, the time-delays at different stages may be different, and therefore the regulation function with multiple time delays $g_{i j}(\cdot)$ is introduced. In many synthetic GRNs, the monotone regulation functions are not just simply added together in practice, but may be coupled with another variable which indicates the relationship between two biochemical substance in GRNs [22,24]. Then, $y_{i j}(\cdot) h_{i j}\left(z_{j}(\cdot)\right)$ is introduced in the model to describe such a complicated property. To ease notation, $h_{i j}(\cdot)$ is also called regulation function, which has the same property with $f_{i j}(\cdot)$ and $g_{i j}(\cdot)$ and we call $y_{i j}(\cdot) h_{i j}\left(z_{j}(\cdot)\right)$ multivariable regulation function. $y_{i j}(\cdot)$ is an element that belongs to $\left[z_{1}(\cdot), z_{2}(\cdot), \ldots, z_{n}(\cdot)\right]^{T}$ but $y_{i j}(\cdot) \neq z_{j}(\cdot)$. Obviously, if $y_{i j}(\cdot)=z_{j}(\cdot), y_{i j}(\cdot) h_{i j}\left(z_{j}(\cdot)\right)$ would have a similar form with $f_{i j}\left(z_{j}(\cdot)\right)$ and the multivariable term can be eliminated. Regulation function is used to capture the combined effect of several regulatory proteins on the control of gene expression or protein degradation and it describes the connection and topology structure of biochemical substance. 
Remark 1: Linear control theory has received great research interest and the corresponding results have been fruitful. However, almost all practical systems are inherently nonlinear. Sometimes, linearization is a powerful tool, but it may prevent us from gaining the insight of nonlinear phenomenon. In neural networks, the structure of the model described by nonlinear differential equations is similar to the GRNs' structure discussed here $[46,47]$. In neural networks, the activation function is like the regulation function in GRNs, but it is just a monotone nonlinear function of only one variable which is totally different from the multivariable regulation functions. The regulation mechanism in GRNs not only follows the "adding" logic like neural networks but also the "coupling" logic. In neural networks, the approach to dealing with nonlinearity is usually based on a "linearization" idea that sets a linear boundary to be an approximation of the nonlinear activation functions. Such an idea works well when there is one variable, but may face unmanageable difficulties when it comes to the multivariable regulation functions. In other words, we are not able to inherit the method for dealing with nonlinear terms in neural networks. Therefore, there is a need to transform the model into a new form so as to facilitate the handling of such nonlinear terms.

In the following, let us consider the regulation functions in order to show the existence of multiple equilibrium points, hence the multistability.

Remark 2: We consider a simplified GRN model:

$$
\dot{z}_{i}(t)=-a_{i} z_{i}(t)+\sum_{j=1}^{n} b_{i j} R_{i j}\left(z_{j}(t)\right) .
$$

A regulation function often found in the literature is the Hill type [14]:

$$
R_{i j}\left(z_{j}\right)=\frac{z_{j}^{H_{i j}}(t)}{\theta_{j}^{H_{i j}}+z_{j}^{H_{i j}}(t)},
$$

with $\theta_{j}>0$ is the threshold for the regulatory influence of $z_{j}$ on a target biochemical substance $z_{i}$, and $H_{i j}$ is the Hill coefficients. The function ranges from 0 to 1 and increases as $z_{j} \rightarrow \infty$, so that an increase in $z_{j}$ will tend to increase the expression rate of the biochemical substance, then biochemical substance $j$ is an activator of gene $i$. If biochemical substance $j$ is an repressor of gene $i$, then

$$
R_{i j}\left(z_{j}\right)=1-\frac{z_{j}^{H_{i j}}(t)}{\theta_{j}^{H_{i j}}+z_{j}^{H_{i j}}(t)} .
$$

Then, the GRN (2) can be rewritten as

$$
\dot{z}_{i}(t)=-a_{i} z_{i}(t)+\sum_{j=1}^{n} b_{i j} \bar{R}_{i j}\left(z_{j}(t)\right)+u_{i}
$$

for $i=1,2, \ldots, n$, with

$$
\bar{R}_{i j}\left(z_{j}(t)\right)=\frac{z_{j}^{H_{i j}}(t)}{\theta_{j}^{H_{i j}}+z_{j}^{H_{i j}}(t)}, u_{i}=\sum_{j \in \mathcal{F}_{i}} \alpha_{i j},
$$

where $\mathcal{F}_{i}$ is the set of all the $j$ which is a repressor of gene $i$, and $u_{i}$ is defined as a basal rate. If $b_{i j}=\alpha_{i j}$, $z_{j}$ is an activator of $z_{i}$; if $b_{i j}=0, z_{j}$ is no link with $z_{i}$; if $b_{i j}=-\alpha_{i j}, z_{j}$ is a repressor of $z_{i}$. If $\theta_{j}=1$, the regulation function is in a standard form. (3) and (4) can be easily transformed to standard form.

Let $\dot{z}_{i}(t)=0$, that is, $a_{i} z_{i}(t)-u_{i}=\sum_{j=1}^{n} b_{i j} \bar{R}_{i j}\left(z_{j}(t)\right)$, whose solution defines the equilibrium point. For convenience, we consider one variable case, i.e., we define right hand of the equality above $f(z)=z^{H_{i j}} /(1+$ 
$z^{H_{i j}}$ ), left hand $h(z)=a z-u$. It is shown that there could be three cross points at most when $H_{i j}=2$ in Fig. 1(a) and two cross points when $H_{i j}=1$ in Fig. 1(b).

(a)

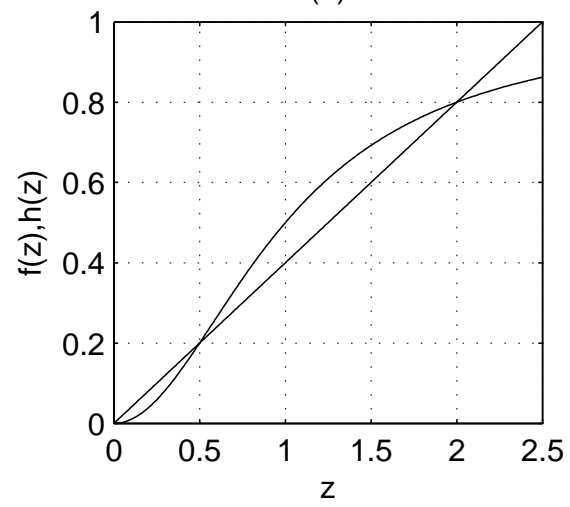

(b)

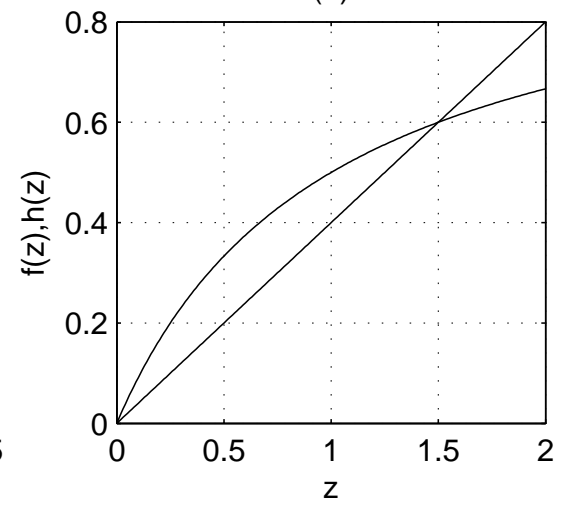

Fig. 1. (a) $f(z)=z^{2} /\left(1+z^{2}\right)$ and $h(z)=0.4 z$. Three equilibrium points can be achieved. (b) $f(z)=z /(1+z)$ and $h(z)=0.4 z$. Two equilibrium points can be obtained.

Assume that a GRN (1) has $N$ equilibrium points and let $z_{k}^{*}=\left(z_{k 1}^{*}, z_{k 2}^{*}, \ldots, z_{k n}^{*}\right)^{T}$ be the $k$ th equilibrium point, $i=1,2, \cdots, N$. So we have

$$
0=-a_{i} z_{k i}^{*}+\sum_{j=1}^{n} b_{i j} f_{i j}\left(z_{k j}^{*}\right)+\sum_{j=1}^{n} c_{i j} g_{i j}\left(z_{k j}^{*}\right)+\sum_{j=1}^{n} d_{i j} y_{k i j}^{*} h_{i j}\left(z_{k j}^{*}\right)+u_{i} .
$$

Subtracting (6) from (1) gives

$$
\begin{aligned}
\dot{z}_{i}(t)-\dot{z}_{k i}^{*}= & -a_{i}\left[z_{i}(t)-z_{k i}^{*}\right]+\sum_{j=1}^{n} b_{i j}\left[f_{i j}\left(z_{j}(t)\right)-f_{i j}\left(z_{k j}^{*}\right)\right] \\
& +\sum_{j=1}^{n} c_{i j}\left[g_{i j}\left(z_{j}\left(t-\tau_{i}\right)\right)-g_{i j}\left(z_{k j}^{*}\right)\right]+\sum_{j=1}^{n} d_{i j}\left[y_{i j}(t) h_{i j}\left(z_{j}(t)\right)-y_{k i j}^{*} h_{i j}\left(z_{k j}^{*}\right)\right] .
\end{aligned}
$$

Let

$$
\begin{aligned}
z_{k i j}(\cdot) & =z_{i j}(\cdot), \\
x_{k i}(\cdot) & =z_{i}(\cdot)-z_{k i}^{*}, \\
y_{k i j}(\cdot) & =y_{i j}(\cdot)-y_{k i j}^{*}, \\
f_{k i j}\left(x_{k j}\right) & =f_{i j}\left(x_{k j}+z_{k j}^{*}\right)-f_{i j}\left(z_{k j}^{*}\right), \\
g_{k i j}\left(x_{k j}\right) & =g_{i j}\left(x_{k j}+z_{k j}^{*}\right)-g_{i j}\left(z_{k j}^{*}\right), \\
h_{k i j}\left(x_{k j}\right) & =h_{i j}\left(x_{k j}+z_{k j}^{*}\right)-h_{i j}\left(z_{k j}^{*}\right), \\
\bar{h}_{k i j}\left(x_{k j}(t)\right) & =z_{k i j}(t) h_{k i j}\left(x_{k j}(t)\right), \\
\sum_{j=1}^{n} d_{i j} h_{i j}\left(z_{k j}^{*}\right) x_{k i j}(t) & =-\sum_{j=1}^{n} e_{k i j} x_{k j}(t) .
\end{aligned}
$$

We have

$$
\dot{x}_{k i}(t)=-a_{i} x_{k i}(t)+\sum_{j=1}^{n} b_{i j} f_{k i j}\left(x_{k j}(t)\right)+\sum_{j=1}^{n} c_{i j} g_{k i j}\left(x_{k j}\left(t-\tau_{i}\right)\right)+\sum_{j=1}^{n} d_{i j} \bar{h}_{k i j}\left(x_{k j}(t)\right)-\sum_{j=1}^{n} e_{k i j} x_{k j}(t) .
$$


For convenience, see Appendix for the derivation.

Equivalently, (16) can be written as

$$
\dot{x}(t)=-\left(A+E_{k}\right) x(t)+\sum_{i=1}^{n} B_{i} f_{k i}(x(t))+\sum_{i=1}^{n} C_{i} g_{k i}\left(x\left(t-\tau_{i}\right)\right)+\sum_{i=1}^{n} D_{i} \bar{h}_{k i}(x(t)),
$$

where

$$
\begin{aligned}
x(t)= & {\left[\begin{array}{c}
x_{k 1}(t) \\
x_{k 2}(t) \\
\vdots \\
x_{k n}(t)
\end{array}\right], f_{k i}(x(t))=\left[\begin{array}{c}
f_{k i 1}\left(x_{k 1}(t)\right) \\
f_{k i 2}\left(x_{k 2}(t)\right) \\
\vdots \\
f_{k i n}\left(x_{k n}(t)\right)
\end{array}\right], } \\
g_{k i}\left(x\left(t-\tau_{i}\right)\right)= & {\left[\begin{array}{c}
g_{k i 1}\left(x_{k 1}\left(t-\tau_{i}\right)\right) \\
g_{k i 2}\left(x_{k 2}\left(t-\tau_{i}\right)\right) \\
\vdots \\
g_{k i n}\left(x_{k n}\left(t-\tau_{i}\right)\right)
\end{array}\right], \bar{h}_{k i}(x(t))=\left[\begin{array}{c}
y_{k i 1}(t) h_{k i 1}\left(x_{k 1}(t)\right) \\
y_{k i 2}(t) h_{k i 2}\left(x_{k 2}(t)\right) \\
\vdots \\
y_{k i n}(t) h_{k i n}\left(x_{k n}(t)\right)
\end{array}\right] . }
\end{aligned}
$$

Consider the regulation function $f_{i j}\left(z_{j}\right)$, which is divided into $k_{f i j \text { max }}$ piecewise intervals along the variable $z_{j}$. If there exist $N$ equilibrium points of (1), we let $k_{f i j \max }=N$ and select $N$ intervals

$$
\Omega_{f}=\left\{\begin{array}{l}
{\left[\xi_{0 i j}, \xi_{1 i j}\right]^{\delta_{1 i j}^{(i)}} \times\left[\xi_{1 i j}, \xi_{2 i j}\right]_{2 i j}^{(i)} \times \cdots \times\left[\xi_{(N-1) i j}, \xi_{N i j}\right]^{\delta_{N i j}^{(i)}},} \\
\left(\delta_{1 i j}^{(i)}, \delta_{2 i j}^{(i)}, \ldots, \delta_{N i j}^{(i)}\right)=(1,0, \ldots, 0) \text { or }(0,1, \ldots, 0), \ldots, \text { or }(0, \ldots, 0,1), i=1,2, \ldots, n
\end{array}\right\} .
$$

Then, the $k$ th equilibrium point lies in the following region

$$
\Omega_{f_{k}}=\left\{\prod_{i=1}^{n}\left[\cap \prod_{j=1}^{n}\left[\xi_{(k-1) i j}, \xi_{k i j}\right]\right]\right\}
$$

Similarly, consider the regulation function $g_{i j}\left(z_{j}\right)$, linear function $r_{i j}\left(z_{i j}(t)\right)=z_{i j}(t)$ and regulation function $h_{i j}\left(z_{j}\right)$, the $k$ th equilibrium point lies in the following regions

$\Omega_{g_{k}}=\left\{\prod_{i=1}^{n}\left[\cap \prod_{j=1}^{n}\left[\lambda_{(k-1) i j}, \lambda_{k i j}\right]\right]\right\}, \Omega_{r_{k}}=\left\{\prod_{i=1}^{n}\left[\cap \prod_{j=1}^{n}\left[\theta_{(k-1) i j}, \theta_{k i j}\right]\right]\right\}, \Omega_{h_{k}}=\left\{\prod_{i=1}^{n}\left[\cap \prod_{j=1}^{n}\left[\mu_{(k-1) i j}, \mu_{k i j}\right]\right]\right\}$

respectively.

Assumption 1: Let $k_{f i j \max }=N$. Each regulation function in $(1), f_{i j}(\cdot), i, j=1,2, \ldots, n$, satisfies the following condition when $\forall x_{k}, y_{k} \in\left[\xi_{i j(k-1)}, \xi_{i j k}\right], x_{k} \neq y_{k}, 1 \leq k \leq N$ :

$$
\alpha_{k i j}^{-} \leq \frac{f_{i j}\left(x_{k}\right)-f_{i j}\left(y_{k}\right)}{x_{k}-y_{k}} \leq \alpha_{k i j}^{+}
$$

where $\alpha_{k i j}^{-}$and $\alpha_{k i j}^{+}$are nonnegative constants.

Because the $k$ th equilibrium point is shifted to origin, the new equilibrium point $\mathbf{0}$ lies in the region

$$
\bar{\Omega}_{f_{k}}=\left\{\prod_{i=1}^{n}\left[\cap \prod_{j=1}^{n}\left[\bar{\xi}_{(k-1) i j}, \bar{\xi}_{k i j}\right]\right]\right\} .
$$

where $\bar{\xi}_{(k-1) i j}=\xi_{(k-1) i j}-z_{k j}^{*}<0, \bar{\xi}_{k i j}=\xi_{k i j}-z_{k j}^{*}>0$. 
By (9), (11) and Assumption 1, it is not difficult to verify that $\forall x_{j} \in\left[\bar{\xi}_{(k-1) i j}, 0\right) \cup\left(0, \bar{\xi}_{k i j}\right], i=1,2, \ldots, n$,

$$
\alpha_{k i j}^{-} \leq \frac{f_{k i j}\left(x_{j}\right)}{x_{j}} \leq \alpha_{k i j}^{+}
$$

and it is easy to see that $f_{k i j}(0)=0$.

Similarly, by (9), (12) and Assumption 1, it is not difficult to verify that $\forall x_{j} \in\left[\bar{\lambda}_{(k-1) i j}, 0\right) \cup\left(0, \bar{\lambda}_{k i j}\right]$, $i=1,2, \ldots, n$,

$$
\beta_{k i j}^{-} \leq \frac{g_{k i j}\left(x_{j}\right)}{x_{j}} \leq \beta_{k i j}^{+}
$$

where $\bar{\lambda}_{(k-1) i j}=\lambda_{(k-1) i j}-z_{k j}^{*}<0, \bar{\lambda}_{k i j}=\lambda_{k i j}-z_{k j}^{*}>0, \beta_{k i j}^{-}$and $\beta_{k i j}^{+}$are nonnegative constants. It is easy to see that $g_{k i j}(0)=0$.

Letting $x_{k i j}^{-}=\theta_{(k-1) i j}$ and $x_{k i j}^{+}=\theta_{k i j}$, we get

$$
x_{k i j}^{-} \leq y_{k i j}(t) \leq x_{k i j}^{+}
$$

Obviously, $x_{k i j}^{-}$and $x_{k i j}^{+}$are positive constants because the concentration of biochemical substance can't be negative.

Similarly, by (9), (13) and Assumption 1, it is not difficult to verify that $\forall x_{j} \in\left[\bar{\mu}_{(k-1) i j}, 0\right) \cup\left(0, \bar{\mu}_{k i j}\right]$, $i=1,2, \ldots, n$,

$$
\sigma_{k i j}^{-} \leq \frac{h_{k i j}\left(x_{j}\right)}{x_{j}} \leq \sigma_{k i j}^{+}
$$

where $\bar{\mu}_{(k-1) i j}=\mu_{(k-1) i j}-z_{k j}^{*}<0, \bar{\mu}_{k i j}=\mu_{k i j}-z_{k j}^{*}>0, \sigma_{k i j}^{-}$and $\sigma_{k i j}^{+}$are nonnegative constants. It is easy to see that $h_{k i j}(0)=0$.

Let $k_{r i j \max }=k_{h i j \max }=N, i, j=1,2, \ldots, n, 1 \leq k \leq N$ and $\gamma_{k i j}^{-}=x_{k i j}^{-} \sigma_{k i j}^{-}, \gamma_{k i j}^{+}=x_{k i j}^{+} \sigma_{k i j}^{+}$. By (20), (21) and Assumption 1, we can have the following corollary.

Corollary 1: Each multivariable regulation function in (17), $y_{k i j} h_{k i j}\left(x_{j}\right), i, j=1,2, \ldots, n$, satisfies the following condition when $\forall x_{j} \in\left[\bar{\lambda}_{i j(k-1)}, 0\right) \cup\left(0, \bar{\lambda}_{i j k}\right], \forall y_{k i j} \in\left[x_{k i j}^{-}, x_{k i j}^{+}\right]$:

$$
\gamma_{k i j}^{-} \leq \frac{y_{k i j} h_{k i j}\left(x_{j}\right)}{x_{j}} \leq \gamma_{k i j}^{+}
$$

Remark 3: The inequalities (18), (19) and (21) are similar to the one proposed in [49,50] for the activation function of neural networks. As pointed out in $[49,50]$, this description could be non-monotonic, and is more general than the usual sigmoid functions and the recently commonly used Lipschitz conditions. We like to point out that such a description is very precise/tight in quantifying the lower and upper bounds of the regulation functions, hence very helpful for using LMI-based approach to reduce the possible conservatism.

\section{Multistability Conditions of GRNs}

In this section, we present our multistability condition for the GRN with multiple time delays and multivariable regulation functions described in the previous section.

Definition 1: A GRN is said to have $N$-stability if it has $N(N \geq 1)$ stable equilibrium points. In this case, the GRN is said to be $N$-stable. Specially, a GRN has bistability if $N=2$. 
Theorem 1: The system in (1) is asymptotically $N$-stable, if there exist matrices $P_{k}>0, Q_{k j}>0, Z_{k j}>0$, and diagonal matrices $U_{k i}^{+}, U_{k i}^{-}, V_{k i}^{+}, V_{k i}^{-}, W_{k i}^{+}$and $W_{k i}^{-}, k=1, \cdots, N, i=1, \cdots, n, j=1,2$, such that the following LMIs hold:

$$
\Theta_{k}+\Lambda_{k}+\Lambda_{k}^{T}<0
$$

where

$$
\begin{aligned}
& \Theta_{k}=\Theta_{k P}+\sum_{i=1}^{n}\left(\Theta_{k Q i}+\Theta_{k Z i}+\Theta_{k U i}+\Theta_{k V i}+\Theta_{k W i}\right), \\
& \Theta_{k P}=M_{k P}^{T} \bar{P}_{k} M_{k P}, \Theta_{k U i}=M_{k U_{i}^{+}}^{T} \bar{U}_{k i}^{+} M_{k U_{i}^{+}}+M_{k U_{i}^{-}}^{T} \bar{U}_{k i}^{-} M_{k U_{i}^{-}}, \\
& \Theta_{k Q i}=M_{k Q_{i}}^{T} \bar{Q}_{k i} M_{k Q_{i}}, \Theta_{k V i}=M_{k V_{i}^{+}}^{T} \bar{V}_{k i}^{+} M_{k V_{i}^{+}}+M_{k V_{i}^{-}}^{T} \bar{V}_{k i}^{-} M_{k V_{i}^{-}}, \\
& \Theta_{k Z i}=M_{k Z_{i}}^{T} \bar{Z}_{k i} M_{k Z_{i}}, \Theta_{k W i}=M_{k W_{i}^{+}}^{T} W_{k i}^{+} M_{k W_{i}^{+}}+M_{k W_{i}^{-}}^{T} \bar{W}_{k i}^{-} M_{k W_{i}^{-}} \text {, } \\
& \Lambda_{k}=X\left[-\left(A+E_{k}\right) \quad-I \quad 0 \sum_{i=1}^{n} B_{i} \sum_{i=1}^{n} C_{i} \sum_{i=1}^{n} D_{i}\right], \\
& \bar{P}_{k}=\left[\begin{array}{cc}
0_{n} & P_{k} \\
P_{k} & 0_{n}
\end{array}\right], M_{k P}=\left[\begin{array}{ccc}
0_{n} & I_{n} & 0_{n, 4 n^{2}} \\
\hline I_{n} & 0_{n,(4 n+1) n}
\end{array}\right],
\end{aligned}
$$

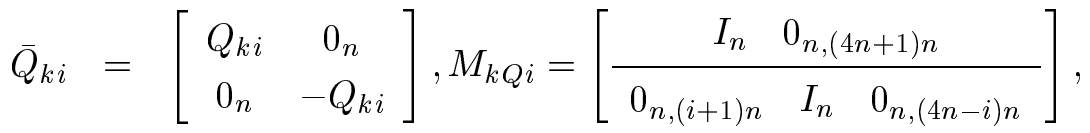

$$
\begin{aligned}
& \bar{Z}_{k i}=\left[\begin{array}{cc}
\tau_{i} Z_{k i} & 0_{n} \\
0_{n} & -1 / \tau_{i} Z_{k i}
\end{array}\right], M_{k Z i}=\left[\begin{array}{cccc} 
& 0_{n} & I_{n} & 0_{n, 4 n^{2}} \\
\hline I_{n} & 0_{n, i n} & -I_{n} & 0_{n,(4 n-i) n}
\end{array}\right] \text {, }
\end{aligned}
$$

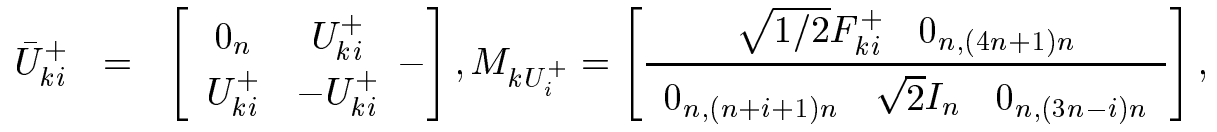

$$
\begin{aligned}
& \bar{U}_{k i}^{-}=\left[\begin{array}{cc}
0_{n} & U_{k i}^{-} \\
U_{k i}^{-} & -U_{k i}^{-}
\end{array}\right], M_{k U_{i}^{-}}=\left[\begin{array}{ccc}
-\sqrt{1 / 2} F_{k i}^{-} & 0_{n,(4 n+1) n} \\
\hline 0_{n,(n+i+1) n} & -\sqrt{2} I_{n} & 0_{n,(3 n-i) n}
\end{array}\right] \text {, } \\
& \bar{V}_{k i}^{+}=\left[\begin{array}{cc}
0_{n} & V_{k i}^{+} \\
V_{k i}^{+} & -V_{k i}^{+}
\end{array}\right], M_{k V_{i}^{+}}=\left[\begin{array}{ccc}
0_{n,(i+1) n} & \sqrt{1 / 2} G_{k i}^{+} & 0_{n,(4 n-i) n} \\
\hline 0_{n,(2 n+i+1) n} & \sqrt{2} I_{n} & 0_{n,(2 n-i) n}
\end{array}\right] \text {, } \\
& \bar{V}_{k i}^{-}=\left[\begin{array}{cc}
0_{n} & V_{k i}^{-} \\
V_{k i}^{-} & -V_{k i}^{-}
\end{array}\right], M_{k V_{i}^{-}}=\left[\begin{array}{ccc}
0_{n,(i+1) n} & -\sqrt{1 / 2} G_{k i}^{-} & 0_{n,(4 n-i) n} \\
\hline 0_{n,(2 n+i+1) n} & -\sqrt{2} I_{n} & 0_{n,(2 n-i) n}
\end{array}\right] \text {, }
\end{aligned}
$$

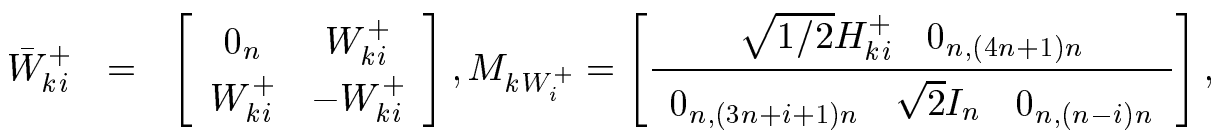

$$
\begin{aligned}
& \bar{W}_{k i}^{-}=\left[\begin{array}{cc}
0_{n} & W_{k i}^{-} \\
W_{k i}^{-} & -W_{k i}^{-}
\end{array}\right], M_{k W_{i}^{-}}=\left[\begin{array}{ccc}
-\sqrt{1 / 2} H_{k i}^{-} & 0_{n,(4 n+1) n} \\
\hline 0_{n,(3 n+i+1) n} & -\sqrt{2} I_{n} & 0_{n,(n-i) n}
\end{array}\right] \text {. }
\end{aligned}
$$

Proof: See Appendix.

If time delay is assumed to be zero, then the GRN becomes:

$$
\dot{z}_{i}(t)=-a_{i} z_{i}(t)+\sum_{j=1}^{n} b_{i j} f_{i j}\left(z_{j}(t)\right)+\sum_{j=1}^{n} b_{i j} z_{i j}(t) h_{i j}\left(z_{j}(t)\right) .
$$


For the $k$ th equilibrium point, by transformation we have

$$
\dot{x}(t)=-\left(A+E_{k}\right) x(t)+\sum_{i=1}^{n} B_{i} f_{k i}(x(t))+\sum_{i=1}^{n} D_{i} \bar{h}_{k i}(x(t)) .
$$

Based on Theorem 1, we can get the following corollary.

Corollary 2: The system in (23) is asymptotically $N$-stable, if there exist matrices $P_{k}>0$, and diagonal matrices $U_{k i}^{+}, U_{k i}^{-}, W_{k i}^{+}$and $W_{k i}^{-}, k=1, \cdots, N, i=1, \cdots, n, j=1,2$, such that the following LMIs hold:

$$
\Theta_{k}+\Lambda_{k}+\Lambda_{k}^{T}<0
$$

where

$$
\begin{aligned}
& \Theta_{k}=M_{k P}^{T} \bar{P}_{k} M_{k P}+\sum_{i=1}^{n}\left(\Theta_{k U i}+\Theta_{k W i}\right), \\
& \Theta_{k U i}=M_{k U_{i}^{+}}^{T} \bar{U}_{k i}^{+} M_{k U_{i}^{+}}+M_{k U_{i}^{-}}^{T} \bar{U}_{k i}^{-} M_{k U_{i}^{-}}, \\
& \Theta_{k W i}=M_{k W_{i}^{+}}^{T} \bar{W}_{k i}^{+} M_{k W_{i}^{+}}+M_{k W_{i}^{-}}^{T} \bar{W}_{k i}^{-} M_{k W_{i}^{-}} \text {, } \\
& \Lambda_{k}=X\left[-\left(A+E_{k}\right) \quad-I \sum_{i=1}^{n} B_{i} \sum_{i=1}^{n} D_{i}\right], \\
& \bar{P}_{k}=\left[\begin{array}{ll}
0_{n} & P_{k} \\
P_{k} & 0_{n}
\end{array}\right], M_{k P}=\left[\begin{array}{ccc}
0_{n} & I_{n} & 0_{n, 2 n^{2}} \\
\hline I_{n} & 0_{n,(2 n+1) n}
\end{array}\right],
\end{aligned}
$$

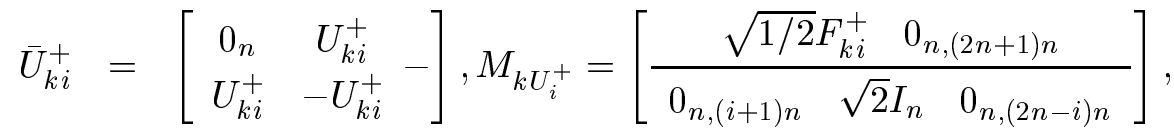

$$
\begin{aligned}
& \bar{U}_{k i}^{-}=\left[\begin{array}{cc}
0_{n} & U_{k i}^{-} \\
U_{k i}^{-} & -U_{k i}^{-}
\end{array}\right], M_{k U_{i}^{-}}=\left[\begin{array}{ccc}
-\sqrt{1 / 2} F_{k i}^{-} & 0_{n,(2 n+1) n} \\
\hline 0_{n,(i+1) n} & -\sqrt{2} I_{n} & 0_{n,(2 n-i) n}
\end{array}\right] \text {, }
\end{aligned}
$$

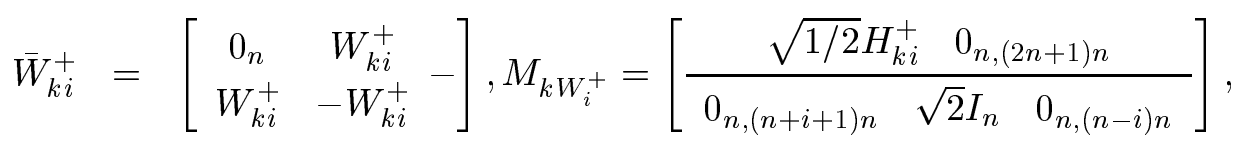

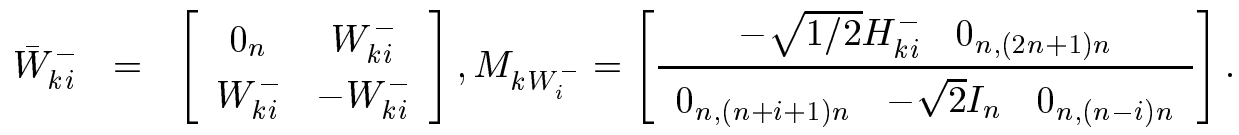

Remark 4: Though not in ordinary form of LMIs, Theorem 1 and Collary 2 are indeed in the standard LMIs form, which can be easily solved by the standard software. Moreover, this form simplified as $W_{X}^{T} X W_{X}+$ $W_{Y}^{T} Y W_{Y}$ is more laconic. It expresses the LMIs in several parts, each of which has a symmetric structure with the matrix variable to be determined in center. Here, $W_{X}$ is the parameter matrix of linear combination of vector elements. For example, $a x_{1}+b x_{2}=0$ could be written as $W_{X} x^{T}=0$, where $W_{X}=\left[\begin{array}{ll}a & b\end{array}\right]$ and $x=\left[\begin{array}{ll}x_{1} & x_{2}\end{array}\right]$.

Remark 5: In our main results, we propose a general sector-like regulation function to derive stability conditions for GRNs with both multiple time-delays and multivariable regulation functions. Up-to-date techniques are utilized for achieving delay dependence to ensure less conservatism. In the next section, the obtained general multistability conditions are demonstrated via two practical examples.

\section{ILlustrative ExAmples}

In this section, two examples are employed to show 1) the generality of our proposed regulation function; and 2) the applicability of our main results for multistability. There are multivariable regulation functions in 
both of the examples. Specifically, Example 1 is concerned with a Cdc2-Cyclin B/Wee1 system and Example 2 is about a reduced model of lactose system.

Example 1. Consider the well-known Cdc2-Cyclin B/Wee1 system in cell cycle described in [24]:

$$
\begin{aligned}
& \dot{x}_{1}=\alpha_{1}\left(1-x_{1}\right)-\frac{\beta_{1} x_{1}\left(v x_{2}\right)^{\gamma_{1}}}{K_{1}+\left(v x_{2}\right)^{\gamma_{1}}}, \\
& \dot{x}_{2}=\alpha_{2}\left(1-x_{2}\right)-\frac{\beta_{2} x_{2} x_{1}^{\gamma_{2}}}{K_{2}+x_{1}^{\gamma_{2}}},
\end{aligned}
$$

where $x_{1}$ denotes active $\operatorname{Cdc} 2, x_{2}$ denotes active Wee1; $\alpha_{1}=\alpha_{2}=1, \beta_{1}=200, \beta_{2}=10$ are rate constants; $K_{1}=30, K_{2}=1$ are Michaelis (saturation) constants; $\gamma_{1}=\gamma_{2}=4$ are Hill coefficients; and $v$ is a coefficient that reflects the strength of the influence of Wee1 on Cdc2-Cyclin B. We select $v=1$ which guarantees the bistability of $(26)$.

(a)

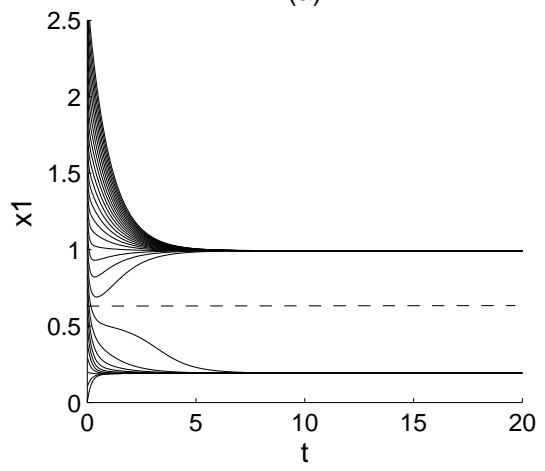

(b)

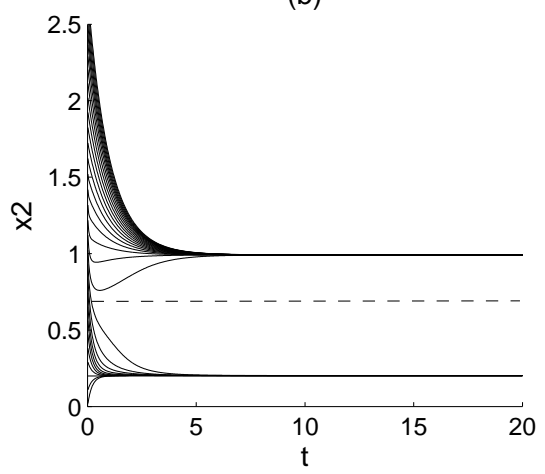

Fig. 2. Transient behavior of system (26).

Simulation results are depicted in Fig. 2. It is shown that two stable states can be achieved. The solid lines represent the stable states and the dashed lines represent the unstable states.

Letting $y_{1}=x_{1}, y_{2}=x_{2} / \sqrt[4]{30}$, we obtain the standard form:

$$
\begin{aligned}
& \dot{y}_{1}=-\alpha_{1} y_{1}-\frac{\beta_{1} y_{1} y_{2}^{4}}{1+y_{2}^{4}}+1, \\
& \dot{y}_{2}=-\alpha_{2} y_{2}-\frac{\beta_{2} y_{2} y_{1}^{4}}{1+y_{1}^{4}}+1 / \sqrt[4]{30},
\end{aligned}
$$

where the regulation function $h(x)=x^{4} /\left(1+x^{4}\right)$, and $d(x)=\dot{h}(x)=4 x^{3} /\left(1+x^{4}\right)^{2} \leq 1.065$. $f(x)$ and $h(x)$ are depicted in Fig. 3. We can get three equilibrium points $x_{1}^{*}=(1,0.17)^{T}, x_{2}^{*}=(0.51,0.62)^{T}$ and $x_{3}^{*}=(0.14,1)^{T}$ of $(26)$, or $y_{1}^{*}=(0.9947,0.0719)^{T}, y_{2}^{*}=(0.51,0.26)^{T}$ and $y_{3}^{*}=(0.1357,0.4258)^{T}$ of $(27)$.

We rewrite model (27) into a compact matrix form

$$
\dot{z}(t)=-\left(A+E_{k}\right) z(t)+D \bar{h}_{k}(z(t))
$$

where

$$
\begin{aligned}
h(z) & =\frac{z^{4}}{1+z^{4}}, z(t)=\left[\begin{array}{c}
z_{1}(t) \\
z_{2}(t)
\end{array}\right]=\left[\begin{array}{l}
y_{1}(t)-y_{k 1}^{*} \\
y_{2}(t)-y_{k 2}^{*}
\end{array}\right], \bar{h}(z(t))=\left[\begin{array}{l}
y_{2}(t) h\left(z_{1}(t)\right) \\
y_{1}(t) h\left(z_{2}(t)\right)
\end{array}\right], \\
A & =\left[\begin{array}{cc}
\alpha_{1} & 0 \\
0 & \alpha_{2}
\end{array}\right], E_{k}=\left[\begin{array}{cc}
\beta_{1} h\left(y_{k 2}^{*}\right) & 0 \\
0 & \beta_{2} h\left(y_{k 1}^{*}\right)
\end{array}\right], D=\left[\begin{array}{cc}
0 & -\beta_{1} \\
-\beta_{2} & 0
\end{array}\right] .
\end{aligned}
$$


When $y_{1}^{*} \in[0.55,+\infty) \times[0,0.25], H_{1}^{+}=\operatorname{diag}\{1.065,0.0620\} \times \operatorname{diag}\{0.25,+\infty\}, H_{1}^{-}=\operatorname{diag}\{0,0\} \times \operatorname{diag}\{0,0.55\}$, we can obtain a feasible solution by solving LMIs with the following obtained matrix variables (for space consideration, we only list the matrix variables $P_{1}, W_{1}^{+}$and $W_{1}^{-}$; and for a valid simulation, we take 100 as a substitute for $+\infty$ in $y_{1}^{*}$ and $\left.H_{1}^{+}\right)$:

$$
P_{1}=\left[\begin{array}{cc}
9.4 & 31.1 \\
31.1 & 9138.3
\end{array}\right], W_{1}^{+}=\left[\begin{array}{cc}
1221.6 & 0 \\
0 & 3584.8
\end{array}\right], W_{1}^{-}=\left[\begin{array}{cc}
67300 & 0 \\
0 & 153330
\end{array}\right] .
$$

When $y_{2}^{*} \in[0.45,0.55] \times[0.25,0.35], H_{2}^{+}=\operatorname{diag}\{0.5586,0.1665\} \times \operatorname{diag}\{0.35,0.5\}, H_{2}^{-}=\operatorname{diag}\{0.3363,0.0620\} \times$ $\operatorname{diag}\{0.25,0.45\}$. The solution is infeasible.

When $y_{3}^{*} \in[0,0.45] \times[0.35,+\infty), H_{3}^{+}=\operatorname{diag}\{0.3363,1.065\} \times \operatorname{diag}\{+\infty, 0.45\}, H_{3}^{-}=\operatorname{diag}\{0,0\} \times \operatorname{diag}\{0.35,0\}$, we can obtain a feasible solution by solving LMIs with the following obtained matrix variables:

$$
P_{2}=\left[\begin{array}{ll}
0.0647 & 0.0878 \\
0.0878 & 2.8020
\end{array}\right], W_{2}^{+}=\left[\begin{array}{cc}
0.0784 & 0 \\
0 & 17.0333
\end{array}\right], W_{2}^{-}=\left[\begin{array}{cc}
127.4051 & 0 \\
0 & 97.9623
\end{array}\right] .
$$

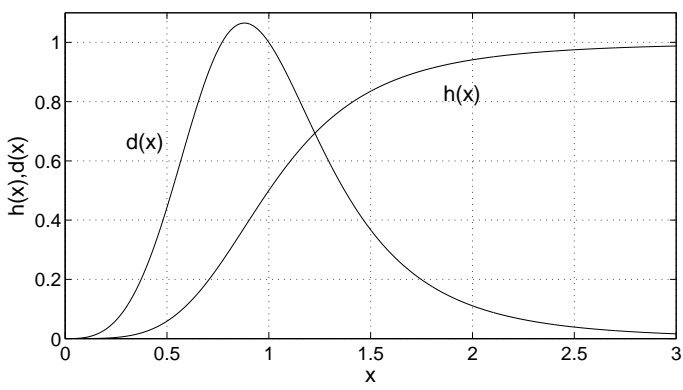

Fig. 3. $h(x)=x^{4} /\left(1+x^{4}\right)$, and $d(x)=4 x^{3} /\left(1+x^{4}\right)^{2}$.

Example 2:. In this example, we consider a reduced model of lactose system. The lac operon consists of a promoter/operator region and three larger structural genes, lacZ, lac Y, and lacA. In the presence of external lactose $(L e)$, lactose is transported into the cell by a permease $(P)$. Intracellular lactose $(L)$ is then broken down into glucose, galactose, and allolactose $(A)$ by the enzyme $\beta$-galactosidase $(B)$. The allolactose $(A)$ feeds back to bind with the lactose repressor and enables the transcription process to proceed. Once the mRNA has been produced, the process of translation is initiated. The lac $\mathrm{Z}$ gene encodes for the mRNA responsible for the production of $\beta$-galactosidase $(B)$ and translation of the lacY gene produces mRNA ultimately responsible for the production of a membrane permease $(P)$.

In [23], it is assumed that there is a constant permease concentration and lactose is in a quasisteady state across the membrane. Therefore, there is a one-to-one relationship between the external and internal lactose. Then lactose $(L)$ and permease $(P)$ dynamics are not considered. A reduced model of three differential equations is considered in [23]:

$$
\begin{aligned}
\dot{A} & =\alpha_{A} B \frac{L}{K_{L}+L}-\beta_{A} B \frac{A}{K_{A}+A}-\tilde{\gamma}_{A} A, \\
\dot{B} & =\alpha_{B} e^{-\mu \tau_{B}} M_{\tau_{B}}-\tilde{\gamma}_{B} B \\
\dot{M} & =\alpha_{M} \frac{1+K_{1}\left(e^{-\mu \tau_{M}} A_{\tau_{M}}\right)^{n}}{K+K_{1}\left(e^{-\mu \tau_{M}} A_{\tau_{M}}\right)^{n}}-\tilde{\gamma}_{M} M,
\end{aligned}
$$


where $M$ is the mRNA concentration, $B$ is the $\beta$ galactosidase concentration, $A$ is the concentration of allolactose (the effector in the lac operon), $L$ is the intracellular lactose concentration, $A_{\tau_{M}} \equiv A\left(t-\tau_{M}\right)$, $M_{\tau_{B}} \equiv M\left(t-\tau_{B}\right)$. and the bacterial growth rate is given by $\mu$. The parameters are given in Table 1 .

Table 1. Parameter values

\begin{tabular}{c|l|l|c|l|l}
\hline parameter & \multicolumn{1}{|c|}{ value } & \multicolumn{1}{c|}{ unit } & parameter & value & unit \\
\hline$n$ & 2 & & $K$ & 7200 & \\
$\mu$ & $3.03 \times 10^{-2}$ & $\min ^{-1}$ & $K_{1}$ & $2.52 \times 10^{-2}$ & $(\mu \mathrm{M})^{-2}$ \\
$\alpha_{M}$ & 997 & $\mathrm{~nm} \cdot \min ^{-1}$ & $K_{L}$ & 0.97 & $\mathrm{mM}$ \\
$\alpha_{B}$ & $1.66 \times 10^{-2}$ & $\mathrm{~min}^{-1}$ & $K_{A}$ & 1.95 & $\mathrm{mM}$ \\
$\alpha_{A}$ & $1.76 \times 10^{4}$ & $\min ^{-1}$ & $\beta_{A}$ & $2.15 \times 10^{4}$ & $\min ^{-1}$ \\
$\gamma_{M}$ & 0.411 & $\min ^{-1}$ & $\tau_{M}$ & 0.10 & $\mathrm{~min}$ \\
$\gamma_{B}$ & $8.33 \times 10^{-4}$ & $\min ^{-1}$ & $\tau_{B}$ & 2.00 & $\mathrm{~min}$ \\
$\gamma_{A}$ & $1.35 \times 10^{-2}$ & $\min ^{-1}$ & & & \\
\hline
\end{tabular}

We rewrite model (28) into a compact matrix form

$$
\dot{x}=-\left(\bar{A}+E_{k}\right) x+\bar{B} f_{k}(x)+\bar{C}_{1} g_{k 1}\left(x_{\tau_{M}}\right)+\bar{C}_{2} g_{k 2}\left(x_{\tau_{B}}\right)+\bar{D} \bar{h}_{k}(x)
$$

where

$$
\begin{aligned}
& g\left(A_{\tau}\right)=\frac{1+K_{1}\left(e^{-\mu \tau} A_{\tau}\right)^{n}}{K+K_{1}\left(e^{-\mu \tau} A_{\tau}\right)^{n}}, h(A)=\frac{A}{K_{A}+A}, \bar{L}=\frac{L}{K_{L}+L} \\
& x=\left[\begin{array}{c}
A_{k} \\
B_{k} \\
M_{k}
\end{array}\right]=\left[\begin{array}{c}
A-A_{k}^{*} \\
B-B_{k}^{*} \\
M-M_{k}^{*}
\end{array}\right], x_{\tau_{M}}=\left[\begin{array}{c}
A_{\tau_{M} k} \\
B_{\tau_{M} k} \\
M_{\tau_{M} k}
\end{array}\right]=\left[\begin{array}{c}
A_{\tau_{M}}-A_{k}^{*} \\
B_{\tau_{M}}-B_{k}^{*} \\
M_{\tau_{M}}-M_{k}^{*}
\end{array}\right], \\
& x_{\tau_{B}}=\left[\begin{array}{c}
A_{\tau_{B} k} \\
B_{\tau_{B} k} \\
M_{\tau_{B} k}
\end{array}\right]=\left[\begin{array}{c}
A_{\tau_{B}}-A_{k}^{*} \\
B_{\tau_{B}}-B_{k}^{*} \\
M_{\tau_{B}}-M_{k}^{*}
\end{array}\right], f_{k}(x(t))=\left[\begin{array}{c}
0 \\
B_{k} \\
0
\end{array}\right] \text {, } \\
& g_{k 1}\left(x_{\tau_{M}}\right)=\left[\begin{array}{c}
g\left(A_{\tau_{M} k}\right) \\
0 \\
M_{\tau_{M} k}
\end{array}\right], g_{k 2}\left(x_{\tau_{B}}\right)=\left[\begin{array}{c}
g\left(A_{\tau_{B} k}\right) \\
0 \\
M_{\tau_{B} k}
\end{array}\right], \bar{h}_{k}(x(t))=\left[\begin{array}{c}
B h\left(A_{k}\right) \\
0 \\
0
\end{array}\right] \text {, } \\
& \bar{A}=\left[\begin{array}{ccc}
\tilde{\gamma}_{A} & 0 & 0 \\
0 & \tilde{\gamma}_{B} & 0 \\
0 & 0 & \tilde{\gamma}_{M}
\end{array}\right], E_{k}=\left[\begin{array}{ccc}
0 & \beta_{A} h\left(A_{k}^{*}\right) & 0 \\
0 & 0 & 0 \\
0 & 0 & 0
\end{array}\right], \bar{B}=\left[\begin{array}{ccc}
0 & \alpha_{A} \bar{L} & 0 \\
0 & 0 & 0 \\
0 & 0 & 0
\end{array}\right], \\
& \bar{C}_{1}=\left[\begin{array}{ccc}
0 & 0 & 0 \\
0 & 0 & 0 \\
\alpha_{M} & 0 & 0
\end{array}\right], \bar{C}_{2}=\left[\begin{array}{ccc}
0 & 0 & 0 \\
0 & 0 & \alpha_{B} e^{-\mu \tau_{B}} \\
0 & 0 & 0
\end{array}\right], \bar{D}=\left[\begin{array}{ccc}
-\beta_{A} & 0 & 0 \\
0 & 0 & 0 \\
0 & 0 & 0
\end{array}\right] \text {. }
\end{aligned}
$$

There are three equilibrium points with $L=50 \mu \mathrm{M}$ :

$$
\begin{aligned}
& S S_{1}^{*}=\left(A_{1}^{*}, B_{1}^{*}, C_{1}^{*}\right)=(4.27 \mu M, 0.23 \mathrm{nM}, 0.46 \mathrm{nM}), \\
& S S_{2}^{*}=\left(A_{2}^{*}, B_{2}^{*}, C_{2}^{*}\right)=(11.73 \mu \mathrm{M}, 0.7 \mathrm{nM}, 1.39 \mathrm{nM}), \\
& S S_{3}^{*}=\left(A_{3}^{*}, B_{3}^{*}, C_{3}^{*}\right)=(64.68 \mu \mathrm{M}, 16.42 \mathrm{nM}, 32.71 \mathrm{nM}),
\end{aligned}
$$


$S S_{1}^{*}$ and $S S_{3}^{*}$ are stable, $S S_{2}^{*}$ is unstable. In the following, we show the effectiveness of our theorem.

When $S S_{1}^{*} \in[0,8] \times[0,0.4] \times[0,1], F_{1}^{+}=\operatorname{diag}\{0,1,0\}, F_{1}^{-}=\operatorname{diag}\{0,1,0\}, G_{11}^{+}=G_{12}^{+}=\operatorname{diag}\{5.5967 \times$ $\left.10^{-5}, 0,1\right\}, G_{11}^{-}=G_{12}^{-}=\operatorname{diag}\{0,0,1\}, H_{1}^{+}=\operatorname{diag}\left\{5.1282 \times 10^{-4}, 0,0\right\} \times \operatorname{diag}\{0.4,0,0\}, H_{1}^{-}=\operatorname{diag}\{5.0864 \times$ $\left.10^{-4}, 0,0\right\} \times \operatorname{diag}\{0,0,0\}$, we can obtain a feasible solution by solving LMIs with the following obtained matrix variables (for space consideration, we only list the matrix variable $P_{1}$ ):

$$
P_{1}=\left[\begin{array}{ccc}
0.0040 & -0.0310 & -0.0006 \\
-0.0310 & 1.2819 & -0.0492 \\
-0.0006 & -0.0492 & 0.0261
\end{array}\right]
$$

When $S S_{2}^{*} \in[8,15] \times[0.4,10] \times[1,15], F_{2}^{+}=\operatorname{diag}\{0,1,0\}, F_{2}^{-}=\operatorname{diag}\{0,1,0\}, G_{21}^{+}=G_{22}^{+}=\operatorname{diag}\{1.0482 \times$ $\left.10^{-4}, 0,1\right\}, G_{21}^{-}=G_{22}^{-}=\operatorname{diag}\left\{5.5967 \times 10^{-5}, 0,1\right\}, H_{2}^{+}=\operatorname{diag}\left\{5.0864 \times 10^{-4}, 0,0\right\} \times \operatorname{diag}\{10,0,0\}, H_{2}^{-}=$ $\operatorname{diag}\left\{5.0502 \times 10^{-4}, 0,0\right\} \times \operatorname{diag}\{0.4,0,0\}$, the solution is infeasible.

When $S S_{3}^{*} \in[15,+\infty) \times[10,+\infty) \times[15,+\infty), F_{3}^{+}=\operatorname{diag}\{0,1,0\}, F_{3}^{-}=\operatorname{diag}\{0,1,0\}, G_{21}^{+}=G_{32}^{+}=$ $\operatorname{diag}\left\{1.215 \times 10^{-3}, 0,1\right\}, G_{31}^{-}=G_{32}^{-}=\operatorname{diag}\{0,0,1\}, H_{3}^{+}=\operatorname{diag}\left\{5.0502 \times 10^{-4}, 0,0\right\} \times \operatorname{diag}\{+\infty, 0,0\}, H_{3}^{-}=$ $\operatorname{diag}\left\{4.6401 \times 10^{-4}, 0,0\right\} \times \operatorname{diag}\{10,0,0\}$, we can obtain a feasible solution by solving LMIs with the following obtained matrix variables (for space consideration, we only list the matrix variables $P_{2}$ ):

$$
P_{2}=\left[\begin{array}{ccc}
0.0029 & 0.0028 & -0.0025 \\
0.0028 & 0.5506 & -0.0344 \\
-0.0025 & -0.0344 & 0.0181
\end{array}\right],
$$

which confirms the bistability of this GRN.

\section{Conclusion}

In this paper, we have made an effort to show the possibility of applying control theory to investigate the multistability of a GRN, therefore having potential applications in the design of synthetic gene circuits with multistability. A novel and uniform mathematical formulation is proposed to describe a GRN with multiple time delays and multivariable regulation functions. A method has been presented for the analysis of multistability of such a GRN. By using a Lyapunov-Krasovskii functional (LKF) approach and linear matrix inequalities (LMIs) techniques, the multistability criteria for a GRN with multiple time delays and multivariable regulation functions have been established in the form of LMIs, which can be readily verified by using standard numerical software. An important feature of the results reported here is that all the multistability conditions are dependent on the delays, which is made possible by utilizing the most updated techniques for achieving delay dependence. Also, our multistability conditions are applicable to several different regulation functions, which cover many types of currently investigated GRNs, especially including the complicated multivariable regulation functions. Two examples have been employed to illustrate the applicability and usefulness of the developed theoretical results, which are concerned with, respectively, a Cdc2-Cyclin B/Wee1 system model and a lactose system model, both of which display bistability. 


\section{APPENDIX}

A. Derivation of (16)

By (7), (8), (9), (10), (11), (12), (13), (14) and (15), we have

$$
\begin{aligned}
& \dot{x}_{k i}(t)=-a_{i} x_{k i}(t)+\sum_{j=1}^{n} b_{i j} f_{k i j}\left(x_{k j}(t)\right)+\sum_{j=1}^{n} c_{i j} g_{k i j}\left(x_{k j}\left(t-\tau_{i}\right)\right) \\
&+\sum_{j=1}^{n} d_{i j}\left[y_{k i j}(t) h_{i j}\left(x_{k j}(t)+z_{k j}^{*}\right)+y_{k i j}^{*} h_{k i j}\left(x_{k j}(t)\right)\right] \\
&=-a_{i} x_{k i}(t)+\sum_{j=1}^{n} b_{i j} f_{k i j}\left(x_{k j}(t)\right)+\sum_{j=1}^{n} c_{i j} g_{k i j}\left(x_{k j}\left(t-\tau_{i}\right)\right) \\
&+\sum_{j=1}^{n} d_{i j}\left[y_{k i j}(t) h_{i j}\left(x_{k j}(t)+z_{k j}^{*}\right)-y_{k i j}(t) h_{i j}\left(z_{k j}^{*}\right)\right] \\
&+\sum_{j=1}^{n} d_{i j} y_{k i j}(t) h_{i j}\left(z_{k j}^{*}\right)+\sum_{j=1}^{n} d_{i j} y_{k i j}^{*} h_{k i j}\left(x_{k j}(t)\right) \\
&=-a_{i} x_{k i}(t)+\sum_{j=1}^{n} d_{i j} y_{k i j}(t) h_{i j}\left(z_{k j}^{*}\right)+\sum_{j=1}^{n} b_{i j} f_{k i j}\left(x_{k j}(t)\right)+\sum_{j=1}^{n} c_{i j} g_{k i j}\left(x_{k j}\left(t-\tau_{i}\right)\right) \\
&+\sum_{j=1}^{n}\left[d_{i j} y_{k i j}(t) h_{k i j}\left(x_{k j}(t)\right)+d_{i j} y_{k i j}^{*}(t) h_{k i j}\left(x_{k j}(t)\right)\right] \\
&+\sum_{j=1}^{n} d_{i j} y_{k i j}(t) h_{k i j}\left(x_{k j}(t)\right) . \\
& x_{k i}(t)+\sum_{j=1}^{n} d_{i j} h_{i j}\left(z_{k j}^{*}\right) y_{k i j}(t)+\sum_{j=1}^{n} b_{i j} f_{k i j}\left(x_{j}(t)\right)+\sum_{j=1}^{n} c_{i j} g_{k i j}\left(x_{j}\left(t-\tau_{i}\right)\right) \\
&
\end{aligned}
$$

Then (16) could be obtained.

\section{B. Proof of Theorem 1}

We first show that the $k$ th equilibrium point is asymptotically stable. The Lyapunov-Krasovskii functional is defined as follows:

$$
\begin{aligned}
V_{k}(x(t)) & =V_{k 1}(x(t))+V_{k 2}(x(t))+V_{k 3}(x(t)), \\
V_{k 1}(x(t)) & =x^{T}(t) P_{k} x(t), \\
V_{k 2}(x(t)) & =\sum_{i=1}^{n} \int_{t-\tau_{i}}^{t} x^{T}(\alpha) Q_{k i} x(\alpha) d \alpha, \\
V_{k 3}(x(t)) & =\sum_{i=1}^{n} \int_{-\tau_{i}}^{0} \int_{t+\beta}^{t} \dot{x}^{T}(\alpha) Z_{k i} \dot{x}(\alpha) d \alpha d \beta .
\end{aligned}
$$


The derivatives of $V_{k j}(x(t)), j=1,2,3$, are given by

$$
\begin{aligned}
\dot{V}_{k 1}(x(t)) & =2 x^{T}(t) P_{k} \dot{x}(t) \\
\dot{V}_{k 2}(x(t)) & =\sum_{i=1}^{n}\left[x^{T}(t) Q_{k i} x(t)-x^{T}\left(t-\tau_{i}\right) Q_{k i} x\left(t-\tau_{i}\right)\right] \\
\dot{V}_{k 3}(x(t)) & =\sum_{i=1}^{n}\left[\tau_{i} \dot{x}^{T}(t) Z_{k i} \dot{x}(t)-\int_{t-\tau_{i}}^{t} \dot{x}^{T}(\alpha) Z_{k i} \dot{x}(\alpha) d \alpha\right] .
\end{aligned}
$$

From Jensen's inequality, we can easily get

$$
\begin{aligned}
& -\int_{t-\tau_{i}}^{t} \dot{x}^{T}(\alpha) Z_{k i} \dot{x}(\alpha) d \alpha \\
\leq & -\frac{1}{\tau_{i}}\left[\int_{t-\tau_{i}}^{t} \dot{x}(\alpha) d \alpha^{T}\right]^{T} Z_{k i}\left[\int_{t-\tau_{i}}^{t} \dot{x}(\alpha) d \alpha\right] \\
= & -\frac{1}{\tau_{i}}\left[x(t)-x\left(t-\tau_{i}\right)\right]^{T} Z_{k i}\left[x(t)-x\left(t-\tau_{i}\right)\right],
\end{aligned}
$$

By (18), for any scalar $u_{k i}^{+} \geq 0$, it is clear that for $i=1, \cdots, n$,

$$
\sum_{j=1}^{n} u_{k i j}^{+} f_{k i j}\left(x_{j}(t)\right)\left[\alpha_{k i j}^{+} x_{j}(t)-f_{k i j}\left(x_{j}(t)\right)\right] \geq 0,
$$

then

$$
\sum_{i=1}^{n} \sum_{j=1}^{n}\left[u_{k i j}^{+} f_{k i j}\left(x_{j}(t)\right) \alpha_{k i j}^{+} x_{j}(t)-u_{k i j}^{+} f_{k i j}\left(x_{j}(t)\right) f_{k i j}\left(x_{j}(t)\right)\right] \geq 0
$$

or equivalently

$$
\sum_{i=1}^{n}\left[f_{k i}(x(t)) U_{k i}^{+} F_{k i}^{+} x(t)-f_{k i}(x(t)) U_{k i}^{+} f_{k i}(x(t))\right] \geq 0 .
$$

Similarly, for any scalars $u_{k i}^{-} \geq 0, v_{k i}^{+} \geq 0$ and $v_{k i}^{-} \geq 0$, we have, respectively,

$$
\begin{gathered}
\sum_{i=1}^{n}\left[f_{k i}(x(t)) U_{k i}^{-} f_{k i}(x(t))-f_{k i}(x(t)) U_{k i}^{-} F_{k i}^{-} x(t)\right] \geq 0 \\
\sum_{i=1}^{n}\left[g_{k i}\left(x\left(t-\tau_{i}\right)\right) V_{k i}^{+} G_{k i}^{+} x\left(t-\tau_{i}\right)-g_{k i}\left(x\left(t-\tau_{i}\right)\right) V_{k i}^{+} g_{k i}\left(x\left(t-\tau_{i}\right)\right)\right] \geq 0,
\end{gathered}
$$

and

$$
\sum_{i=1}^{n}\left[g_{k i}\left(x\left(t-\tau_{i}\right)\right) V_{k i}^{-} g_{k i}\left(x\left(t-\tau_{i}\right)\right)-g_{k i}\left(x\left(t-\tau_{i}\right)\right) V_{k i}^{-} G_{k i}^{-} x\left(t-\tau_{i}\right)\right] \geq 0 .
$$

Also, by Corollary 1 , for any scalars $w_{k i}^{+} \geq 0$ and $w_{k i}^{-} \geq 0$, we have that for $i=1, \cdots, n$,

$$
\sum_{i=1}^{n}\left[\bar{h}_{k i}(x(t)) W_{k i}^{+} H_{k i}^{+} x(t)-\bar{h}_{k i}(x(t)) W_{k i}^{+} \bar{h}_{k i}(x(t))\right] \geq 0
$$

and

$$
\sum_{i=1}^{n}\left[\bar{h}_{k i}(x(t)) W_{k i}^{-} \bar{h}_{k i}(x(t))-\bar{h}_{k i}(x(t)) W_{k i}^{-} H_{k i}^{-} x(t)\right] \geq 0
$$


In addition, based on (17), for any matrices $X$, we have

$$
\zeta_{k}(t) X\left[-\left(A+E_{k}\right) x(t)+\sum_{i=1}^{n} B_{i} f_{k i}(x(t))+\sum_{i=1}^{n} C_{i} g_{k i}\left(x\left(t-\tau_{i}\right)\right)+\sum_{i=1}^{n} D_{i} \bar{h}_{k i}(x(t))-\dot{x}(t)\right]=0,
$$

with

$$
\zeta_{k}(t)=\left[\begin{array}{c}
x(t) \\
\dot{x}(t) \\
\chi(t-\tau) \\
f_{k}(x(t)) \\
g_{k}(x(t-\tau)) \\
\bar{h}_{k}(x(t))
\end{array}\right]
$$

where

$$
\begin{aligned}
\chi(t-\tau)= & {\left[\begin{array}{c}
x\left(t-\tau_{1}\right) \\
x\left(t-\tau_{2}\right) \\
\vdots \\
x\left(t-\tau_{n}\right)
\end{array}\right], f_{k}(x(t))=\left[\begin{array}{c}
f_{k 1}(x(t)) \\
f_{k 2}(x(t)) \\
\vdots \\
f_{k n}(x(t))
\end{array}\right], } \\
g_{k}(x(t-\tau))= & {\left[\begin{array}{c}
g_{k 1}\left(x\left(t-\tau_{1}\right)\right) \\
g_{k 2}\left(x\left(t-\tau_{2}\right)\right) \\
\vdots \\
g_{k n}\left(x\left(t-\tau_{n}\right)\right)
\end{array}\right], \bar{h}_{k 1}(x(t))=\left[\begin{array}{c}
\bar{h}_{k 2}(x(t)) \\
\vdots \\
\bar{h}_{k n}(x(t))
\end{array}\right] . }
\end{aligned}
$$

By using (17) and (A8)-(A15), we have

$$
\begin{aligned}
\dot{V}_{k}(x(t)) \leq & 2 x^{T}(t) P_{k} \dot{x}(t) \\
& +\sum_{i=1}^{n}\left[x^{T}(t) Q_{k i} x(t)-x^{T}\left(t-\tau_{i}\right) Q_{k i} x\left(t-\tau_{i}\right)\right] \\
& +\sum_{i=1}^{n}\left\{\tau_{i} \dot{x}^{T}(t) Z_{k i} \dot{x}(t)-\frac{1}{\tau_{i}}\left[x(t)-x\left(t-\tau_{i}\right)\right]^{T} Z_{k i}\left[x(t)-x\left(t-\tau_{i}\right)\right]\right\} \\
& +\sum_{i=1}^{n}\left[2 f_{k i}(x(t)) U_{k i}^{+} F_{k i}^{+} x(t)-2 f_{k i}(x(t)) U_{k i}^{+} f_{k i}(x(t))\right] \\
& +\sum_{i=1}^{n}\left[2 f_{k i}(x(t)) U_{k i}^{-} f_{k i}(x(t))-2 f_{k i}(x(t)) U_{k i}^{-} F_{k i}^{-} x(t)\right] \\
& +\sum_{i=1}^{n}\left[2 g_{k i}\left(x\left(t-\tau_{i}\right)\right) V_{k i}^{+} G_{k i}^{+} x\left(t-\tau_{i}\right)-2 g_{k i}\left(x\left(t-\tau_{i}\right)\right) V_{k i}^{+} g_{k i}\left(x\left(t-\tau_{i}\right)\right)\right] \\
& +\sum_{i=1}^{n}\left[2 g_{k i}\left(x\left(t-\tau_{i}\right)\right) V_{k i}^{-} g_{k i}\left(x\left(t-\tau_{i}\right)\right)-2 g_{k i}\left(x\left(t-\tau_{i}\right)\right) V_{k i}^{-} G_{k i}^{-} x\left(t-\tau_{i}\right)\right] \\
& +\sum_{i=1}^{n}\left[2 \bar{h}_{k i}(x(t)) W_{k i}^{+} H_{k i}^{+} x(t)-2 \bar{h}_{k i}(x(t)) W_{k i}^{+} \bar{h}_{k i}(x(t))\right] \\
& +\sum_{i=1}^{n}\left[2 \bar{h}_{k i}(x(t)) W_{k i}^{-} \bar{h}_{k i}(x(t))-2 \bar{h}_{k i}(x(t)) W_{k i}^{-} H_{k i}^{-} x(t)\right]
\end{aligned}
$$




$$
\begin{aligned}
& +2 \zeta_{k}(t) X\left[-\left(A+E_{k}\right) x(t)+\sum_{i=1}^{n} B_{i} f_{k i}(x(t))+\sum_{i=1}^{n} C_{i} g_{k i}\left(x\left(t-\tau_{i}\right)\right)\right. \\
& \left.+\sum_{i=1}^{n} D_{i} \bar{h}_{k i}(x(t))-\dot{x}(t)\right] \\
= & \zeta_{k}^{T}(t)\left(\Theta_{k}+\Lambda_{k}+\Lambda_{k}^{T}\right) \zeta_{k}(t),
\end{aligned}
$$

which follows from $(22)$ that $\dot{V}_{k}(x(t))<-\epsilon_{k}\|x(t)\|^{2}$ for a sufficiently small $\epsilon_{k}>0, k=1, \cdots, N$ and $x(t) \neq 0$, then the asymptotic $N$-stability is established and the GRN (1) is $N$-stable.

\section{REFERENCES}

[1] H. Kitano, Systems Biology: A Brief Overview, Science, vol. 295, no. 5560, pp. 1662-1664, 2002.

[2] H. Kitano, Computational systems biology, Nature, vol. 420, no. 6912, pp. 206-210, 2002.

[3] M. Kirschner, The Meaning of Systems Biology, Cell, vol. 121, no. 4, pp. 503-504, 2005.

[4] S. Benner and A. Sismour, Synthetic biology, Nature Reviews Genetics, vol. 6, no. 7, pp. 533.543, 2005.

[5] D. Endy, Foundations for engineering biology, Nature, vol. 438, no. 7067, pp. 449-453, 2005.

[6] E. Andrianantoandro, S. Basu, D. Karig, and R. Weiss, Synthetic biology: new engineering rules for an emerging discipline, Mol. Syst. Biol, vol. 2, no. 2006.0028, 2006.

[7] M. Elowitz and S. Leibler, A synthetic oscillatory network of transcriptional regulators, Nature, vol. 403, no. 6767, pp. 335-8, 2000 .

[8] T. Gardner, C. Cantor, and J. Collins, Construction of a genetic toggle switch in Escherichia coli. Nature, vol. 403, no. 6767, pp. 339-42, 2000.

[9] J. Hasty, D. McMillen, and J. Collins, Engineered gene circuits, Nature, vol. 420, no. 6912, pp. 224-230, 2002.

[10] L. You, R. Cox III, and R. Weiss, Programmed population control by cell- cell communication and regulated killing, Nature, vol. 428, pp. 868-871, 2004.

[11] R. Weiss, T. Knight Jr, and G. Sussman, Cellular Computation and Communication Using Engineered Genetic Regulatory Networks, In: Cellular Computing, Edited by M. Amos, Oxford University Press, pp. 120-147, 2004.

[12] E. Ozbudak, M. Thattai, H. Lim, B. Shraiman, and A. van Oudenaarden, Multistability in the lactose utilization network of Escherichia coli, Nature, vol. 427, no. 6976, pp. 737-740, 2004.

[13] P. Smolen, D. Baxter, and J. Byrne, Mathematical Modeling of Gene Networks, Neuron, vol. 26, no. 3, pp. 567-580, 2000.

[14] H. de Jong, Modeling and Simulation of Genetic Regulatory Systems: A Literature Review, Journal of Computational Biology, vol. 9, no. 1, pp. 67-103, 2002.

[15] Y. Sun, G. Feng, and J. Cao, Stochastic stability of Markovian switching genetic regulatory networks, Physics Letters A, vol. 373, no. 18-19, pp. 1646-1652, 2009.

[16] W. He and J. Cao, Robust stability of genetic regulatory networks with distributed delay, Cognitive Neurodynamics, vol. 2, no. 4, pp. 355-361, 2008.

[17] M. Xiao and J. Cao, Genetic oscillation deduced from Hopf bifurcation in a genetic regulatory network with delays, Mathematical Biosciences, vol. 215, no. 1, pp. 55-63, 2008.

[18] H. Gao and C. Wang, Delay-dependent Robust $H_{\infty}$ and $L_{2}-L_{\infty}$ filtering for a class of uncertain nonlinear time-delay systems,. IEEE Transactions on Automatic Control, vol. 48, no. 9, pp. 1661-1666, 2003.

[19] H. Gao and C. Wang, A delay-dependent approach to robust $H_{\infty}$ filtering for uncertain discrete-time state-delayed systems. IEEE Transactions on Signal Processing, vol. 52, no. 6, pp. 1631-1640, 2004.

[20] Z. Wang, Y. Liu, M. Li, and X. Liu, Stability analysis for stochastic Cohen-Grossberg neural networks with mixed time delays, IEEE Transactions on Neural Networks, vol. 17, no. 3, pp. 814-820, 2006.

[21] Z. Wang, F. Yang, D. Ho, and X. Liu, Robust $H_{\infty}$ filtering for stochastic time-delay systems with missing measurements, IEEE Transactions on Signal Processing, vol. 54, no. 7, pp. 2579-2587, 2006.

[22] N. Yildirim and M. Mackey, Feedback Regulation in the Lactose Operon: A Mathematical Modeling Study and Comparison with Experimental Data, Biophysical Journal, vol. 84, no. 5, pp. 2841-2851, 2003.

[23] N. Yildirim, M. Santillán, D. Horike, and M. Mackey, Dynamics and bistability in a reduced model of the lac operon, Chaos: An Interdisciplinary Journal of Nonlinear Science, vol. 14, p. 279, 2004.

[24] D. Angeli, J. Ferrell Jr, and E. Sontag, Detection of multistability, bifurcations, and hysteresis in a large class of biological positive-feedback systems, Proceedings of the National Academy of Sciences, vol. 101, no. 7, pp. 1822-1827, 2004. 
[25] C. Li, L. Chen, and K. Aihara, Stability of Genetic Networks With SUM Regulatory Logic: Lur'e System and LMI Approach, IEEE Transactions on Circuits and Systems I: Regular Papers, vol. 53, no. 11, pp. 2451-2458, 2006.

[26] Z. Wang, H. Gao, J. Cao, and X. Liu, On Delayed Genetic Regulatory Networks With Polytopic Uncertainties: Robust Stability Analysis, IEEE Transactions on NanoBioscience, vol. 7, no. 2, pp. 154-163, 2008.

[27] J. Cao and F. Ren, Exponential Stability of Discrete-Time Genetic Regulatory Networks With Delays, IEEE Transactions on Neural Networks, vol. 19, no. 3, pp. 520-523, 2008.

[28] J. Ferrell Jr and E. Machleder, The Biochemical Basis of an All-or-None Cell Fate Switch in Xenopus Oocytes. Science, vol. 280, no. 5365, p. 895, 1998 .

[29] W. Xiong and J. Ferrell Jr, A positive-feedback-based 'bistable memory' module that governs a cell fate decision, Nature, vol. 426, pp. 460-465, 2003.

[30] J. Pomerening, E. Sontag, and J. Ferrell Jr, .Building a cell cycle oscillator: hysteresis and bistability in the activation of Cdc 2, Nature Cell Biology, vol. 5, no. 4, pp. 346-351, 2003.

[31] M. Laurent and N. Kellershohn, Multistability: a major means of differentiation and evolution in biological systems, Trends in Biochemical Sciences, vol. 24, no. 11, pp. 418-422, 1999.

[32] W. Sha, J. Moore, K. Chen, A. Lassaletta, C. Yi, J. Tyson, and J. Sible, Hysteresis drives cell-cycle transitions in Xenopus laevis egg extracts, Proceedings of the National Academy of Sciences, vol. 100, no. 3, pp. 975-980, 2003.

[33] D. Angeli and E. Sontag, Multi-stability in monotone input output systems, Systems E Control Letters, vol. 51, no. 1-2, pp. 185-202, 2004.

[34] D. Angeli and E. Sontag, Monotone control systems, IEEE Transactions on Automatic Control, vol. 48, no. 10, pp. 1684-1698, 2003.

[35] M. Chaves, T. Eissing, and F. Allgower, Bistable Biological Systems: A Characterization Through Local Compact Input-toState Stability, IEEE Transactions on Automatic Control, vol. 53, no. 1, pp. 87-100, 2008.

[36] R. Thomas, D. Thieffry, and M. Kaufman, Dynamical behaviour of biological regulatory networks-I. Biological role of feedback loops and practical use of the concept of the loop-characteristic state, Bulletin of Mathematical Biology, vol. 7, no. 2, pp. 247-276, 1995.

[37] H. Gao and T. Chen, "New results on stability of discrete-time systems with time-varying state delay," IEEE Transactions on Automatic Control, vol. 52, no. 2, pp. 328-334, Feb. 2007.

[38] H. Gao, T. Chen, J. Lam, A new delay system approach to network-based control', Automatica, vol. 44, no. 1, pp. 39-52, 2008.

[39] H. Gao, T. Chen, Network Based $H_{\infty}$ Output Tracking Control. IEEE Transactions on Automatic Control, vol. 53, no. 3, pp. 655-667, 2008.

[40] Z. Wang, Y. Liu, and X. Liu, On global asymptotic stability of neural networks with discrete and distributed delays, Physics Letters A, vol. 345, no. 4-6, pp. 299-308, 2005.

[41] Z. Wang, Y. Liu, M. Li and X. Liu, Stability analysis for stochastic Cohen-Grossberg neural networks with mixed time delays, IEEE Transactions on Neural Networks, vol. 17, pp. 814, May 2006.

[42] J. Cao, G. Feng and Y. Wang, Multistability and multiperiodicity of delayed Cohen-Grossberg neural networks with a general class of activation functions, Physica D, vol. 237, no. 13, pp. 1734-1749, 2008.

[43] X. Nie and J. Cao, Multistability of multitime scale competitive neural networks with time-varying and distributed delays, Nonlinear Analysis, Series B, vol. 10, no. 2, pp. 928-942, 2009.

[44] J.K. Hale, The theory of functional differential equations, Springer-Verlag, New York, 1977.

[45] S. Boyd, L. El Ghaoui, E. Feron, and V. Balakrishnan, Linear Matrix Inequalities in System and Control Theory. Society for Industrial Mathematics, 1994.

[46] J. Hopfield, Neurons with Graded Response Have Collective Computational Properties like Those of Two-State Neurons, Proceedings of the National Academy of Sciences, vol. 81, no. 10, pp. 3088-3092, 1984.

[47] L. Chua and L. Yang, Cellular neural networks: theory, IEEE Transactions on Circuits and Systems, vol. 35, no. 10, pp. $1257-1272,1988$.

[48] J. Tyson and H. Othmer, The dynamics of feedback control circuits in biochemical pathways, Progress in theoretical biology, Vol. 5, 1-62, p. 5, 1978.

[49] Y. Liu, Z. Wang, and X. Liu, Global exponential stability of generalized recurrent neural networks with discrete and distributed delays, Neural Networks, vol. 19, no. 5, pp. 667-675, 2006.

[50] Z. Wang, H. Shu, Y. Liu, D. Ho, and X. Liu, Robust stability analysis of generalized neural networks with discrete and distributed time delays, Chaos, Solitons and Fractals, vol. 30, no. 4, pp. 886-896, 2006. 